\title{
Genomic sequencing of Troides aeacus nucleopolyhedrovirus (TraeNPV) from golden birdwing larvae (Troides aeacus formosanus) to reveal defective Autographa californica NPV genomic features
}

Yu-Feng Huang ${ }^{1}$, Tzu-Han Chen ${ }^{1}$, Zih-Ting Chang ${ }^{2}$, Tai-Chuan Wang ${ }^{3}$, Se Jin Lee ${ }^{4}$, Jong Cheol Kim ${ }^{4}$ Jae Su Kim , Kuo-Ping Chiu ${ }^{1}$ and Yu-Shin Nai ${ }^{*}$

\begin{abstract}
Background: The golden birdwing butterfly (Troides aeacus formosanus) is a rarely observed species in Taiwan. Recently, a typical symptom of nuclear polyhedrosis was found in reared T. aeacus larvae. From the previous Kimura-2 parameter (K-2-P) analysis based on the nucleotide sequence of three genes in this isolate, polh, lef-8 and lef-9, the underlying virus did not belong to any known nucleopolyhedrovirus (NPV) species. Therefore, this NPV was provisionally named "TraeNPV". To understand this NPV, the nucleotide sequence of the whole TraeNPV genome was determined using next-generation sequencing (NGS) technology.

Results: The genome of TraeNPV is 125,477 bp in length with 144 putative open reading frames (ORFs) and its GC content is $40.45 \%$. A phylogenetic analysis based on the 37 baculoviral core genes suggested that TraeNPV is a Group I NPV that is closely related to Autographa californica nucleopolyhedrovirus (AcMNPV). A genome-wide analysis showed that TraeNPV has some different features in its genome compared with other NPVs. Two novel ORFs (Ta75 and Ta139), three truncated ORFs ( $p c n a$, he65 and bro) and one duplicated ORF (38.7 K) were found in the TraeNPV genome; moreover, there are fewer homologous regions (hrs) than there are in AcMNPV, which shares eight hrs within the TraeNPV genome. TraeNPV shares similar genomic features with AcMNPV, including the gene content, gene arrangement and gene/genome identity, but TraeNPV lacks 15 homologous ORFs from AcMNPV in its genome, such as ctx, host cell-specific factor 1 (hcf-1), PNKJPNL, vp15, and apsup, which are involved in the auxiliary functions of alphabaculoviruses.

Conclusions: Based on these data, TraeNPV would be clarified as a new NPV species with defective AcMNPV genomic features. The precise relationship between TraeNPV and other closely related NPV species were further investigated. This report could provide comprehensive information on TraeNPV for evolutionary insights into butterfly-infected NPV.
\end{abstract}

Keywords: Troides aeacus, Troides aeacus nucleopolyhedrovirus, TraeNPV

\footnotetext{
*Correspondence: ysnai@nchu.edu.tw

${ }^{5}$ Department of Entomology, National Chung Hsing University, Taichung,

Taiwan

Full list of author information is available at the end of the article
}

C The Author(s). 2019 Open Access This article is distributed under the terms of the Creative Commons Attribution 4.0 International License (http://creativecommons.org/licenses/by/4.0/), which permits unrestricted use, distribution, and reproduction in any medium, provided you give appropriate credit to the original author(s) and the source, provide a link to the Creative Commons license, and indicate if changes were made. The Creative Commons Public Domain Dedication waiver (http://creativecommons.org/publicdomain/zero/1.0/) applies to the data made available in this article, unless otherwise stated. 


\section{Background}

The golden birdwing butterfly, Troides aeacus formosanus (Rothschild) (Lepidoptera: Papilionidae), is one subspecies of five known $T$. aeacus; it is distributed throughout tropical areas and is also endemic to Taiwan [1]. Golden birdwing butterflies have a large body size and a wingspan that exceeds $15 \mathrm{~cm}$ [2]. The population of the golden birdwing butterfly has been declining due to commercial activity and a loss of habitat fitness, i.e., a loss of host plants [1,3]. Therefore, this butterfly species is protected by the Convention on International Trade in Endangered Species of Wild Fauna and Flora (CITES), and the public will have to make more effort in the conservation management of the $T$. aeacus formosanus population [1]. From our previous investigation, a liquefaction symptom was found in the population of rearing golden birdwing butterfly larvae, and this symptom was similar to that of nuclear polyhedrosis. Polyhedral inclusion bodies (PIBs) were observed, and they filled in the body fluid of moribund larvae. A positive signal indicating a polyhedrin gene fragment was detected by PCR. Apparently, the polyhedrosis of the golden birdwing butterfly larvae is caused by nucleopolyhedrovirus (NPV) infection [4].

There are four genera in the Baculoviridae, including Alphabaculovirus (lepidopteran-specific nucleopolyhedrovirus, NPV), Betabaculovirus (lepidopteran-specific granulovirus), Gammabaculovirus (hymenopteran-specific NPV) and Deltabaculovirus (dipteran-specific NPV) [5]. The phylogenetic analysis based on the polyhedrin (polh) genes could further divide the lepidopteran-specific NPVs into group, I and II [6]. To date, more than 78 complete NPV genomes have been deposited in the NCBI GenBank, and most of them are lepidopteran-specific NPVs. However, the occurrence of NPV epizootics in butterfly species is uncommon. Among these sequenced NPV genomes, only Catopsilia pomona NPV (CapoNPV) was reported as a butterfly-infecting NPV, and it was clarified as a distinct species in Group I Alphabaculovirus [7].

To understand the NPV from the golden birdwing butterfly larvae, the Kimura 2-parameter (K-2-P) distances between the alignment of the polh, lef- 8 and lef- 9 nucleotide sequences were performed as described by Jehle et al. for baculovirus identification and species classification [8]. According to the analysis of K-2-P distances from these three genes, this NPV belongs to the group I baculoviruses and is highly closely related to the Autographa californica nucleopolyhedrovirus (AcMNPV) group [4]. However, most of the distances between this NPV and other closely related NPVs were higher than 0.015 . The K-2-P results also showed an ambiguous taxonomic position for this virus; therefore, the taxonomic status of this virus still requires further clarification. Thus far, we could conclude that this NPV belongs to neither the BmNPV group nor the AcMNPV group. Therefore, this NPV was provisionally named "TraeNPV" [4].

As aforementioned, we attempted to sequence the whole genome of TraeNPV. Furthermore, a phylogenetic analysis based on 37 baculovirus core genes of 77 sequenced baculoviruses will be analysed to clarify the TraeNPV taxonomic issue. The genomic features of the whole genome, including the gene structure, orientations and genome density will be described in this report. Comparative genomic analyses were also performed, and the genome sequences were further compared in detail with the previously published group I NPV type species including AcMNPV [9], Bombyx mori NPV (BmNPV) [10], Maruca vitrata MNPV (MaviMNPV) [11], group II NPV type species LdMNPV [12] and one Betabaculovirus, the Cydia pomonella granulosis virus (CpGV) [13]. This report provides new insight into evolutionary aspects of butterfly-infecting NPVs. Therefore, the precise relationship between TraeNPV and other closely related NPV species could be further investigated.

\section{Results and discussion}

\section{General characteristics of the TraeNPV genome}

The TraeNPV genome is $125,477 \mathrm{bp}$ in length and has a $\mathrm{G}+\mathrm{C}$ content of $40.35 \%$ (see Additional file 1: Table S1). The complete genomic sequence with gene annotation information was submitted to GenBank (accession number: MH077961). The open reading frames (ORFs) were predicted according to the initial criteria for further study. A total of 144 ORFs were identified for further analysis (Fig. 1; Additional file 1: Table S2), and the nucleotides in the TraeNPV genome were numbered sequentially, beginning with the A (designated position 1) of the polyhedrin start codon (ATG). The arrows indicate the directions of the transcripts. The ratio of the ORF orientations was approximately 1:1.06 [clockwise (70/144): anticlockwise (74/144)] for those oriented clockwise with respect to the orientation of the polh gene (ORF1) [14]. The TraeNPV genome had a high number of ORFs, which ranked 18.99\% (15/79) compared to the other 78 sequenced baculovirus genomes (Additional file 2: Figure S1). Among these putative ORFs, $40.97 \%$ (59 ORFs) showed overlap in the genome, and the length of the overlap ranged from $1 \mathrm{bp}$ to 158 bp. Four pairs of ORFs that had a larger overlap than that found in TraeNPV were identified, namely, Ta59 (lef-3)/Ta60 (ac68), Ta72 (ac81)/Ta73 (tlp20), Ta106 (ac121)/Ta107 (ac122) and Ta5 (38.7K)/Ta6 (lef-1). Ta59 overlaps with Ta60 by 52 aa in the opposite ORF direction. Ta72 overlaps with Ta73 by ca. 50 aa. There were ca. 36 aa of overlap between the ORFs Ta106/ Ta107 and Ta5/Ta6. There were 37 conserved genes in 


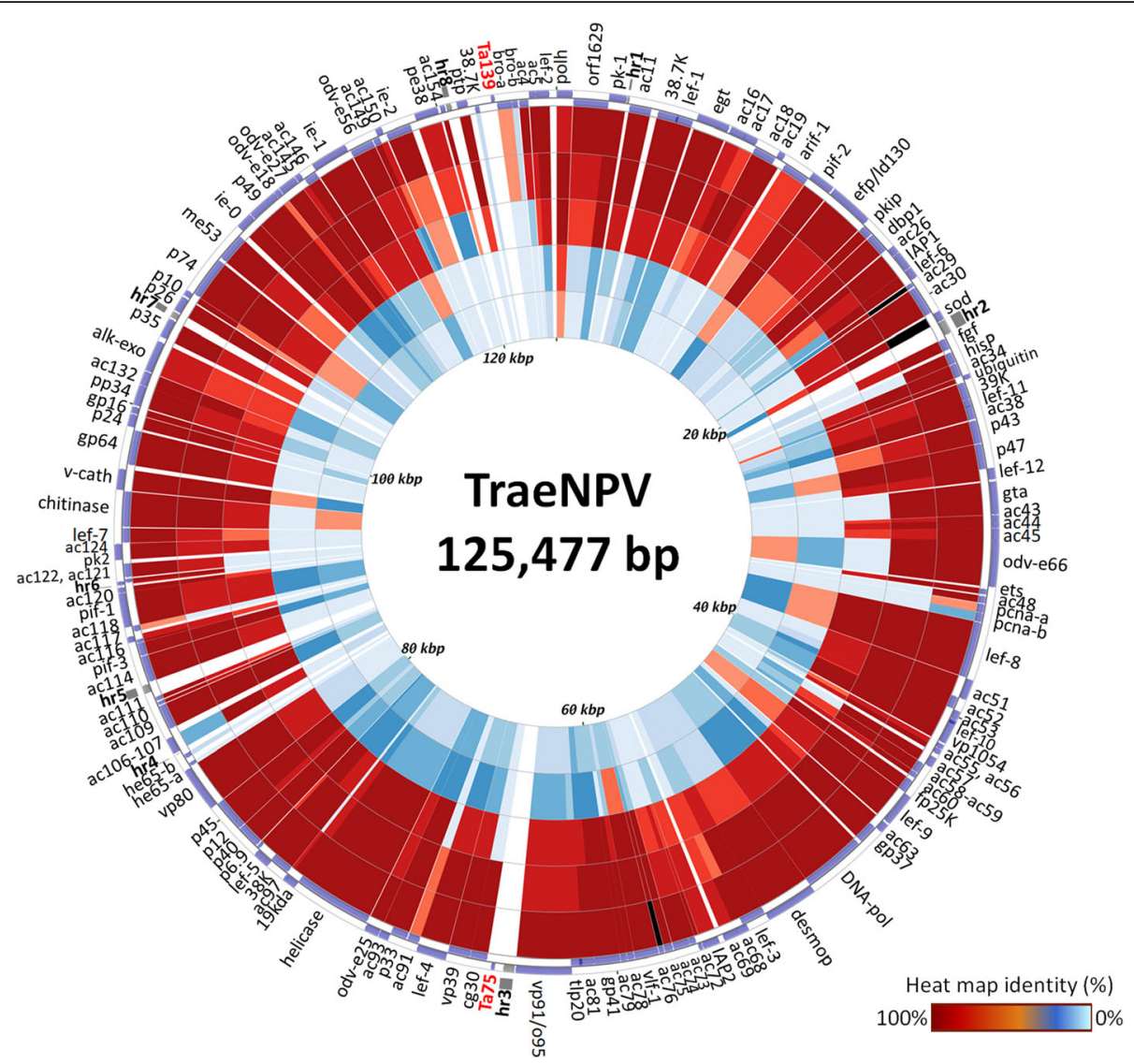

Fig. 1 Genomic circular map and heat map identity of the TraeNPV. The heat map identity of the species AcMNPV, BmNPV, MaviMNPV, LdMNPV and CPGV compared to the orthologous ORFs of TraeNPV are shown on the inner rings in sequence. The darker the red is, the higher the correlated ORF identity. The positions for these $144 \mathrm{ORFs}$, which are listed in Additional file 1: Table S2, are presented as arrowheads with the direction of the arrowhead indicating the orientation of each ORF. The locations for the eight homologous repeat regions (hrs) are indicated

all the baculovirus genomes, including the dipteran and hymenopteran baculoviruses [15-18], and all of these genes were found in the TraeNPV genome. Except in the TraeNPV genome, the Ac108 was found in all the alpha- and betabaculovirus genomes [19]. Moreover, two baculovirus-repeated ORFs (the bro genes bro- $a$ and bro- $a$ ) were also identified in this genomic sequence. Most of the 144 TraeNPV ORFs had related homologues in other baculoviruses except for two unique ORFs (Ta75 and Ta139), which were identified in the TraeNPV genome (Fig. 1; Additional file 1: Table S2).

In addition to these 144 predicted ORFs, other internal spaces were made up of intergenic spaces and common DNA non-coding functional elements (nfes), i.e., homologous regions ( $h r s)$. The TraeNPV genome exhibited $8 h r(h r 1 \sim 8)$ (Fig. 1; Additional file 1: Table S2), and the orientations of the hrs were similar to those of AcMNPV. A conserved non-protein-coding genomic element (CNE, $156 \mathrm{bp}$ ), which was identified as a member of the Alphabaculovirus genus and was speculated to play a role in viral replication, was also found in the TraeNPV genome [20]. The CNE of TraeNPV is located from $118,740 \mathrm{bp}$ to $118,895 \mathrm{bp}$. For the CNE structures, the seven conserved nucleotide clusters $(\mathrm{C} 1 \sim \mathrm{C} 7)$ in the $\mathrm{CNE}$ were also found in the CNE of TraeNPV. According to the structure and nucleotide composition, the conserved nucleotide clusters could also be further divided into dyad symmetry elements (DSs) and TAT-containing sequences (Fig. 2a). In the CNE of TraeNPV, three inverted repeats (IR) are presented in the DS left (DSl), DS central (DSc) and DS right (DSr) regions (Fig. 2a). Regarding the orientation of the CNE in TraeNPV, the location of the CNE lacked any ORF overlap in the TraeNPV genome; by contrast, the AcMNPV CNE overlapped with Ac152 (Fig. 2a). The identity of the CNE showed the highest shared sequence identity (96\%) with that of AcMNPV, while the sequence composition of the TraeNPV CNE (AT content 73.8\%) revealed a higher AT content than that of AcMNPV (AT content $68.6 \%$ ). 


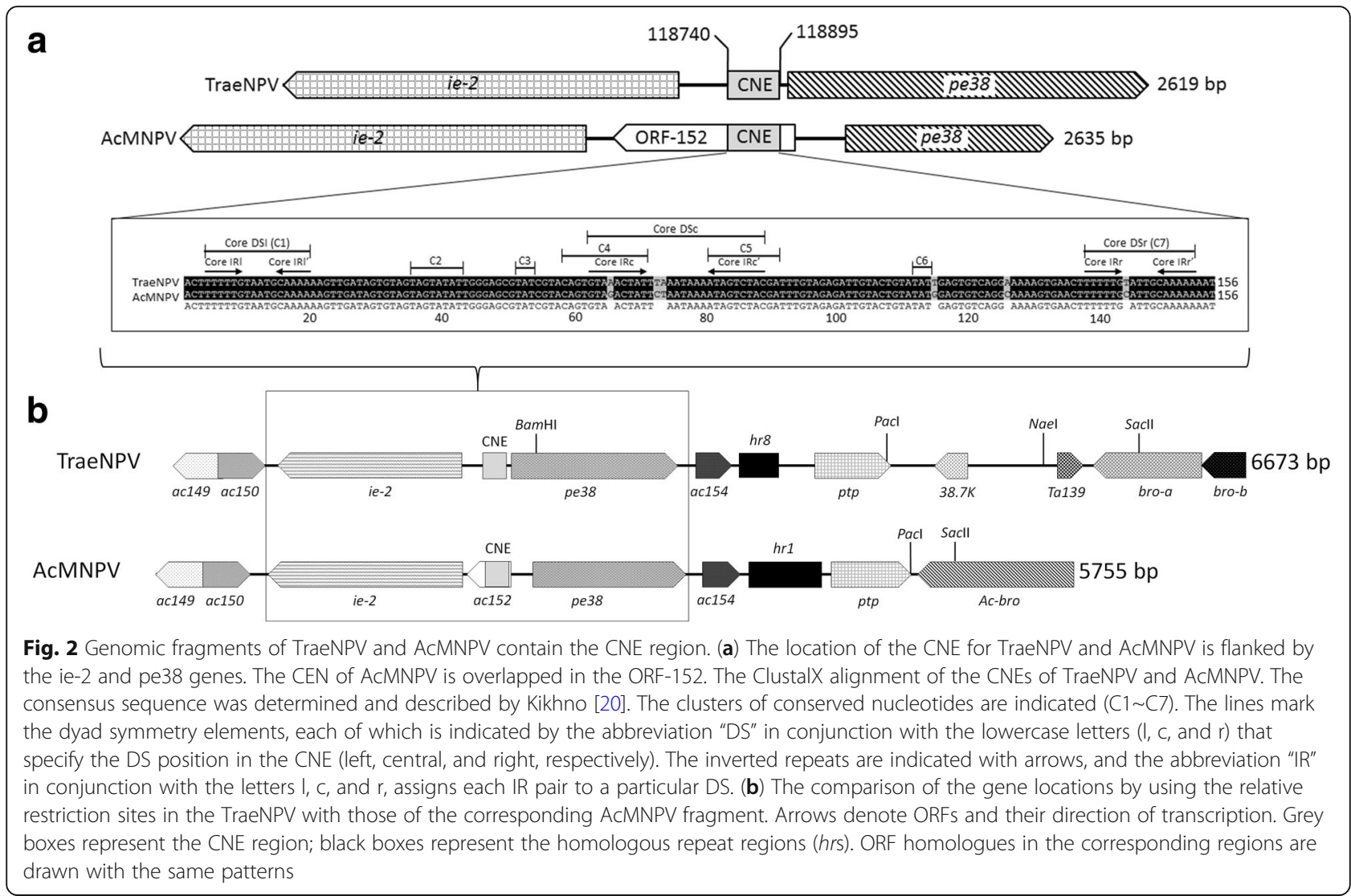

Based on the experimental data obtained using a CNE-deficient AcMNPV bacmid, the CNE was demonstrated to be a polyfunctional genomic element involved in an essential role in AcMNPV pathogenesis [20]. Moreover, it also demonstrated that the CNE position would not impact the function of the CNE, suggesting that the CNE of TraeNPV might share a similar pathogenesis ability.

Taxonomic position and phylogenetic analysis of TraeNPV The phylogenetic analyses of TraeNPV were performed using NJ and ML methods, and the results were inferred from a data set that combined the amino acid sequences of the 37 baculovirus core genes from 77 whole genomic sequenced baculoviruses (Additional file 1: Table S3) [5, 16]. Both of the phylogenetic trees showed a similar result, and the ML trees revealed higher bootstrap values and are shown in Fig. 3. The family Baculoviridae consists of five major clades, the NPVs infecting Lepidoptera (including groups I and II), the GVs, the hymenopteran-specific NPVs, and CuniNPV. This analysis reflected the current systematic assignment of the viruses. Moreover, two subclades within lepidopteran NPV group I resembled the AcMNPV and OpMNPV. The result also indicated that TraeNPV was grouped together with AcMNPV (Fig. 3).
From our previous data, although we attempted to clarify the classification of TraeNPV and its closely related NPVs by K-2-P analysis based on the sequences of polh, lef-8 and lef-9, TraeNPV apparently had an ambiguous relationship with its closely related viral species. The results revealed that TraeNPV belonged to the group I baculoviruses and was highly closely related to the BmMNPV and AcMNPV groups [4]. By contrast, the distances for polh between TraeNPV and the PlxyNPV, RoNPV, AcMNPV groups exceeded the thresholds of the different viral species, and for all the concatenated polh/ lef-8/lef-9 sequences, the distances were apparently much greater than the threshold of the same viral isolates; therefore, the limited data indicate an ambiguous situation for TraeNPV $[4,8]$.

From the comparative genomic studies, the conservation of the general mechanisms underlying baculoviral biology could be speculated; thus, the 37 core genes shared by all the sequenced baculovirus genomes might not only represent the similar function in the mode of viral infection, but they could also reflect the most realistic taxonomic position $[20,21]$. Through the whole genome sequencing and the phylogenetic analysis based on 37 baculoviral core genes, it was revealed that TraeNPV is closely related to AcMNPV rather than BmNPV. 


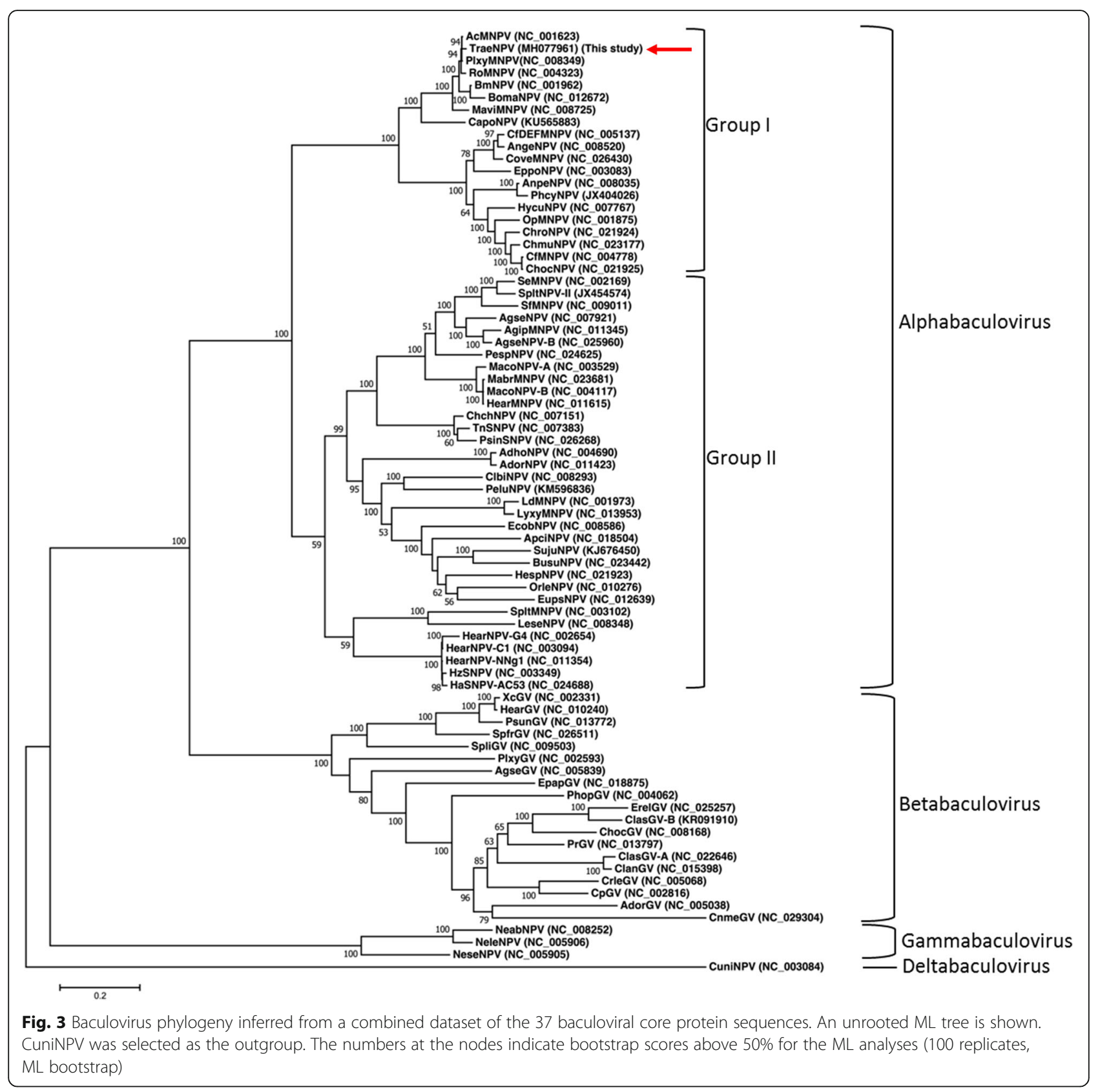

\section{Genome-wide comparisons}

Comparisons of whole genomes and the gene arrangements of the selected ORFs were performed with CGView, Mauve and a gene parity plot analysis. For the whole genome comparisons, TraeNPV showed a highly similar genomic fragment identity compared to AcMNPV and BmNPV, while a lower shared genomic identity was found between TraeNPV and MaviNPV (Additional file 3: Figure S2). In addition, compared to the TraeNPV genome, there are three locations flanked by the ORFs of Ta22/Ta24, Ta74/Ta76 and Ta132/Ta141, which showed a lower shared identity with those of other baculoviruses (Additional file 3: Figure S2). A graphical interpretation of the homologous blocks in viral genomes from alphabaculoviruses from group I and II and from CpGV is shown in Fig. 4. This information also revealed that the conserved segments appeared to be internally free from the genome rearrangement of other baculoviruses; however, a locally collinear block (LCB) deletion between alk-exo (Ta118) and p35 (Ta119) was found in TraeNPV (Fig. 4). Moreover, the gene arrangement of the TraeNPV genome was highly collinear with that of AcMNPV, BmNPV and MaviNPV. For the gene parity plot analysis, the gene arrangement of the TraeNPV genome showed lower collinearity with LdMNPV and CpGV and the ORFs displayed a much more dispersed pattern (Fig. 5). 


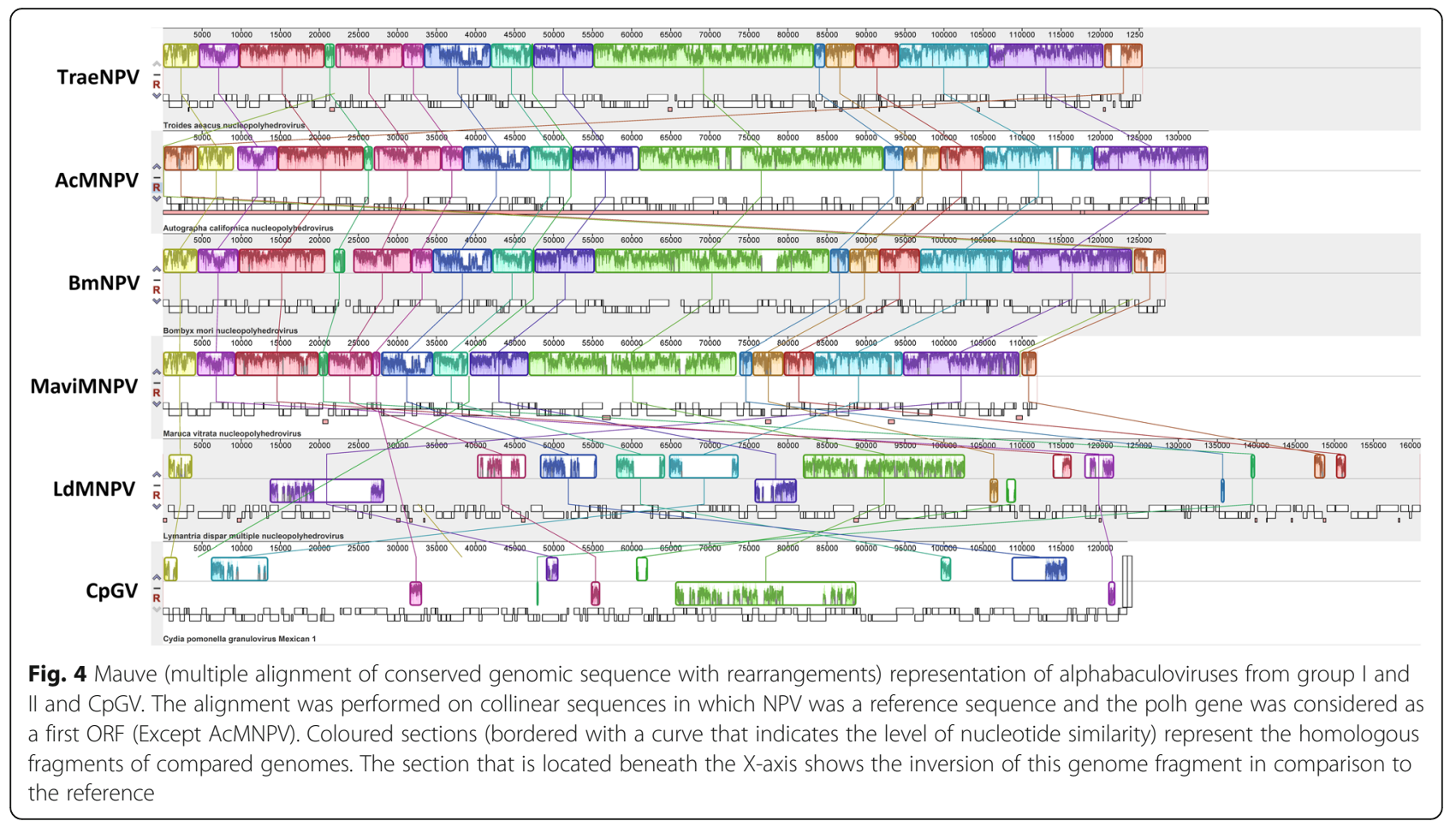

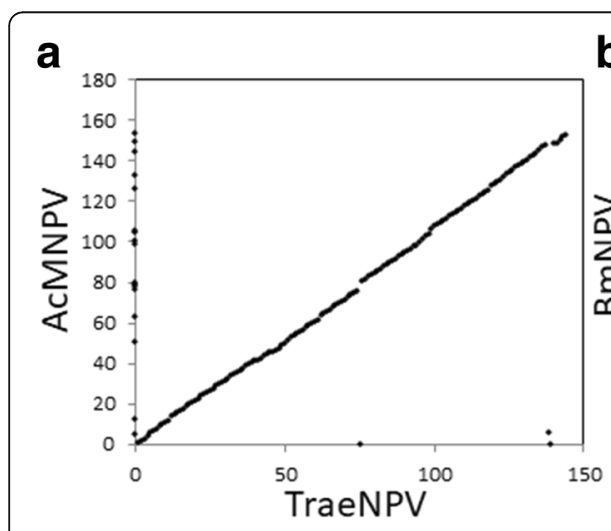

d

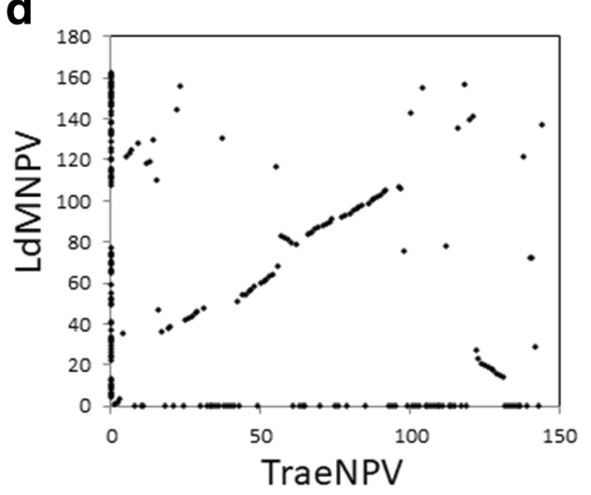

b c
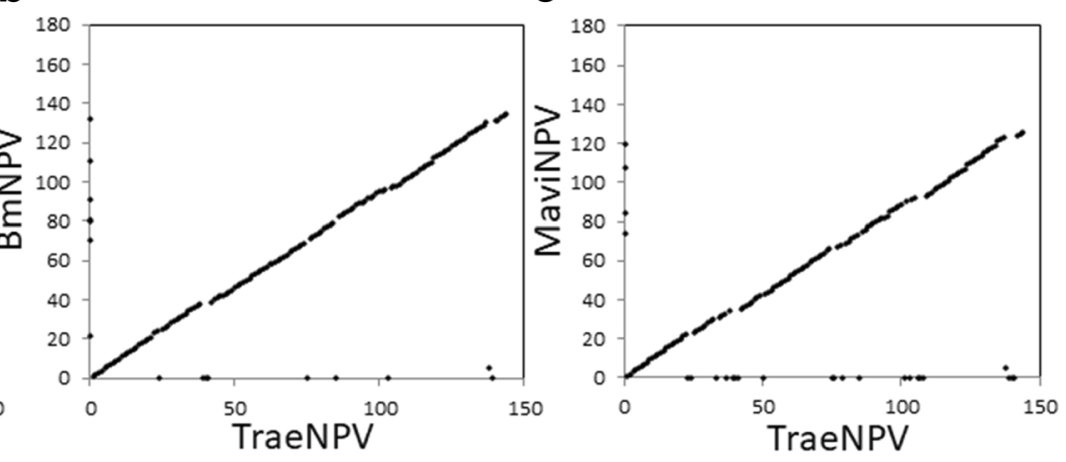

e

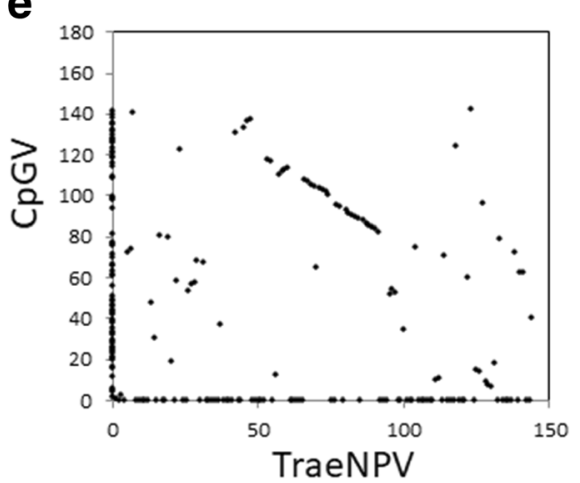

Fig. 5 Gene parity plot analysis of TraeNPV in comparison with (a) AcMNPV, (b) BmNPV, (c) MaviNPV, (d) LdMNPV and (e) CpGV, as indicated. Axes: the relative position of each ORF; dots: ORFs 
A further comparison of the genomic fragments from Ta132 to Ta141 to that of AcMNPV revealed a 1576 bp DNA fragment insertion from the nucleotide positions $121,403 \mathrm{bp}$ to $122,979 \mathrm{bp}$ in the TraNPV genome (Fig. 2b). Within the inserted DNA fragment, one novel gene (Ta139) and one duplicate gene were found; in addition, the restriction enzyme profile also revealed a difference in the Ta132/Ta141 fragment relative to that of AcMNPV (Fig. 2b). Although TraeNPV was similar to AcMNPV and BmNPV in terms of gene organization, the presence of a different region was found upon genome-wide analysis.

According to the comparative analysis of baculoviral genomes, baculoviruses are highly diverse in terms of their GC content, genome length, gene content, and gene organization. These characteristics could reflect the evolutionary history of baculoviruses in adapting to different hosts [21, 22]. Based on the gene content (two novel ORFs were found in TraeNPV and were lacking 15 AcMNPV homologous ORFs) and genomic length (shorter than AcMNPV), TraeNPV might be distinct from AcMNPV.

\section{Comparison of TraeNPV ORFs with other baculoviruses}

TraeNPV shares 142 ORFs with AcMNPV, 136 ORFs with BmNPV, 124 ORFs with MaviMNPV, 90 with LdMNPV and 74 with CpGV. The average shared amino acid sequence identity between TraeNPV and AcMNPV, BmNPV, MaviMNPV, LdMNPV and CpGV were 90.96, $86.61,78.71,33.20$, and $25.61 \%$, respectively. Based on the presented data, TraeNPV is closely related to AcMNPV; of the 142 ORFs that are common to TraeNPV and AcMNPV, only 2 ORFs sharing 100\% identity and 97 ORFs sharing > 95\% identity were found. Of the other 43 ORFs, 18 ORFs sharing 95-90\% identity, 12 ORFs sharing $89-80 \%$ identity and 13 ORFs sharing $<80 \%$ identity were found. It is notable that there were three ORFs, Ta95 (Ac106-107), Ta103 (Ac118) and Ta126 (odv-e18), that had low shared identities $(39,52$ and $61 \%$, respectively) compared to those of the AcMNPV homologues due to the variations in the amino acid lengths, suggesting that there might be amino acid variations between TraeNPV and AcMNPV. In fact, the further analysis showed that variations were found in the amino acid lengths and identities between TraeNPV, AcMNPV and BmNPV (Figs. 1 and 6; Additional file 1: Table S2). In addition, it also showed clear amino acid length differences compared to those of MaviMNPV, LdMNPV and CpGV.

TraeNPV lacks 15 ORFs in AcMNPV and 7 ORFs in BmNPV (Table 1). In addition, there are two pairs of adjacent AcMNPV ORFs (Ac58/Ac59 and Ac106/Ac107) that were fused together into single ORFs (Ta51 and Ta95, respectively) in TraeNPV. As reported for
Rachiplusia ou MNPV-R1, the re-sequencing of these regions in AcMNPV-C6 indicated that the ORF pairs occurred as a single ORF in the AcMNPV-C6 stock [23]. Homologues of these ORFs were also found in other baculovirus genomes in which they were fused into a single ORF (Additional file 1: Table S2).

\section{TraeNPV structural genes}

TraeNPV contains 35 baculovirus structural genes, which were listed by Hayakawa et al. (2000), Jehle et al. (2006) and Thumbi et al. (2013) [5, 21, 24], and only the p15 (Ac87) gene was absent from the TraeNPV genome (Table 2). Of the 35 structural proteins, the P74 protein is associated with occluded virions and is required for oral infectivity $[25,26]$; the VP1054 protein is required for AcMNPV nucleocapsid formation [27]; the P10 protein has been shown to be involved in the formation and stability of polyhedra and may influence cell lysis late in infection [28-30]; VP80 is associated with both ODV and BV in AcMNPV and OpMNPV [31, 32]; and ORF1629 is associated with the basal end of nucleocapsids and is essential for AcMNPV viability [33, 34]. The GP64 protein is the envelope fusion protein of the budded virus, and it is specific to the group I NPVs $[35,36]$. Another envelope fusion protein that is functionally analogous to the GP64 protein called Ld130 is present in all lepidopteran and dipteran baculoviruses that have been completely sequenced, including those that contain gp64. The TraeNPV genome also contains these proteins, and it encodes both GP64 (Ta113) and Ld130 (Ta14). It has been suggested that Ld130 homologues may play a role in the ancient envelope fusion protein, and its fusion function was substituted by gp64; the co-existence of this gene with gp64 might occur because it has other essential functions [36]. There are several genes that encode capsid-associated proteins ( $v p 39$ and $v p 91$ ), ODV envelope proteins (odv-e18, $-e 25,-e 56$, and -e66), DNA binding protein (p6.9), and the tegument protein (gp41) that is also associated with BV production $[37,38]$. Most of these structural genes have highly shared identities in AcMNPV, specifically $>95 \%$ shared identities, suggesting that the structure of the TraeNPV may be similar to that of AcMNPV. The structural protein of TraeNPV shared a high similarity with that of AcMNPV, and there are four structural genes with slightly lower shared identifies with AcMNPV, namely, polh (Ta1; 88\%), gp64 (Ta113; 92\%), odv-e18 (Ta126; 61\%) and odv-e26 (Ta8; 89\%) (Additional file 1: Table S2). It has been reported that the AcMNPV polh consists of a mosaic of group I and group II NPV-specific sequences and it has a chimerical structure [39]. Interestingly, a low shared identity (88\%) for polh was found between TraeNPV and AcMNPV, suggesting that this difference may be related to a process in baculovirus evolution. 

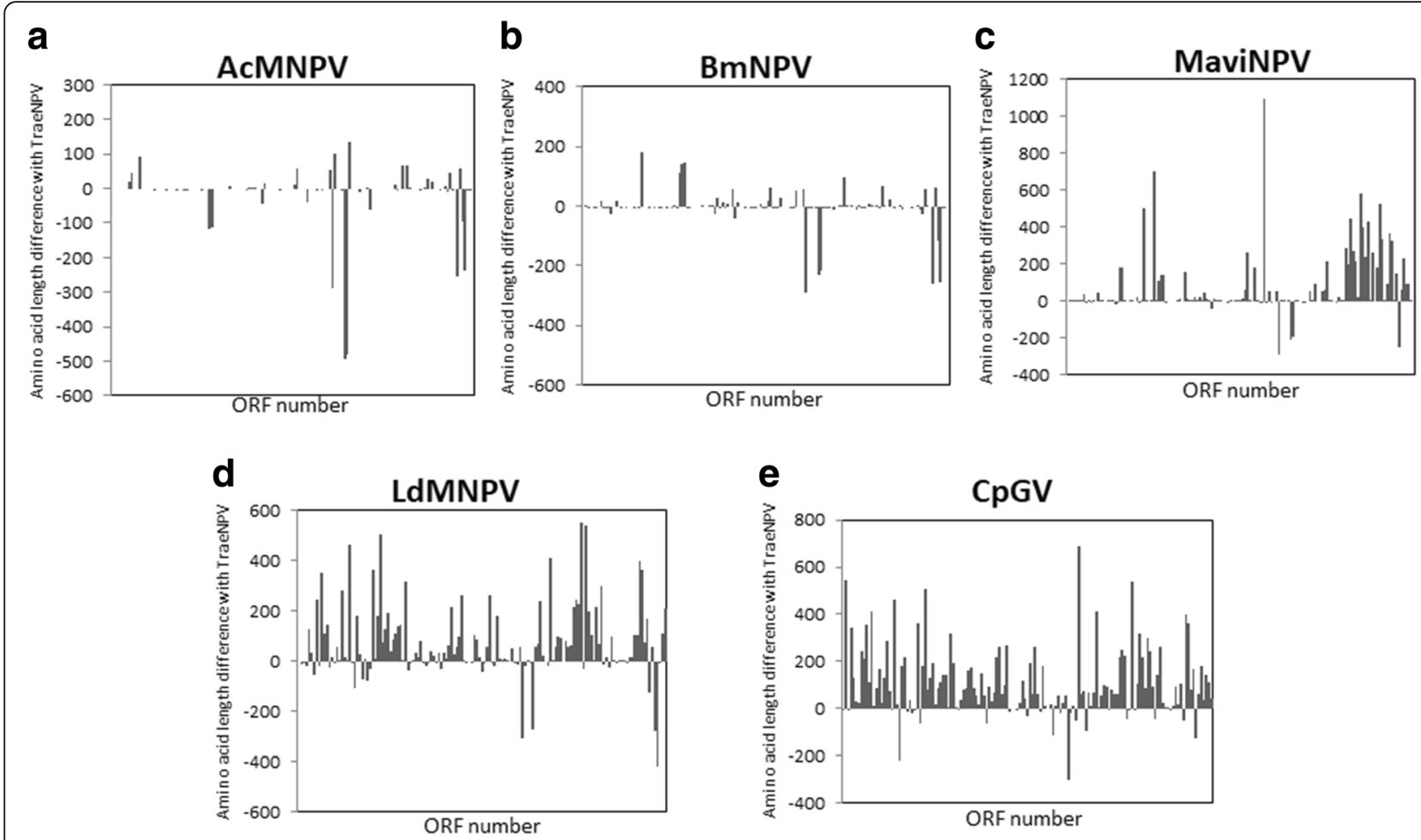

Fig. 6 Amino acid length difference for TraeNPV compared to (a) AcMNPV, (b) BmNPV, (c) MaviNPV, (d) LdMNPV and (e) CpGV, as indicated. Xaxis: the relative position of each ORF; Y-axis dots: amino acid differences

Table 1 AcMNPV and BmNPV ORFs with no homologues in the TraeNPV genome

\begin{tabular}{|c|c|c|c|c|c|}
\hline \multicolumn{3}{|l|}{ AcMNPV } & \multicolumn{3}{|l|}{ BmNPV } \\
\hline${ }^{\mathrm{a} O R F}$ & Length (aa) & Name & $\bar{a} \mathrm{ORF}$ & Length (aa) & Name \\
\hline 3 & 53 & $c t x$ & 22 & 317 & bro-a \\
\hline 7 & 201 & orf603 & 70 (Ac87) & 126 & vp15 \\
\hline 12 & 217 & & 80 & 239 & bro-b \\
\hline 20 & 69 & & 81 & 318 & bro-c \\
\hline 58 & 57 & & 91 (Ac108) & 105 & \\
\hline 70 & 290 & hcf-1 & 111 (Ac134) & 70 & \\
\hline 84 & 188 & & 132 & 241 & bro-e \\
\hline 85 & 53 & & & & \\
\hline 86 & 694 & PNKJPNL & & & \\
\hline $87(B m 70)$ & 126 & vp15 & & & \\
\hline 108 (Bm91) & 105 & & & & \\
\hline $112-113$ & 256 & apsup & & & \\
\hline $134(B m 111)$ & 803 & & & & \\
\hline 140 & 60 & & & & \\
\hline 152 & 92 & & & & \\
\hline
\end{tabular}

${ }^{\mathrm{a} O R F s}$ in the brackets are homologues ORFs between AcMNPV and BmNPV

\section{Transcription-specific genes}

A total of 13 genes involved in late baculovirus gene transcription that are all present on other baculovirus genomes $[5,21,24]$ are also present in the TraeNPV genome, including lef 4-12, $39 \mathrm{~K}, \mathrm{p} 47, \mathrm{vlf}-1$ and pe38 (Table 2). Of these genes, 10 genes (lef-4 - 6, $-8 \sim-12$, $39 k$, and $p 47)$ are required for optimal levels of late gene transcription in the AcMNPV genome [40, 41]. These 10 proteins play a role in the virus-encoded RNA polymerase that recognizes a late promoter element, RTAAG $(\mathrm{R}=\mathrm{A}, \mathrm{T}$, or $\mathrm{G})$ [42]. Moreover, lef-4, lef-8, lef-9, and $p 47$ form a minimal complex with late polymerase activity [43]. Additionally, a conserved gene, vlf-1 might, regulates very late gene transcription and may be involved in DNA processing [44-46]. These genes had high shared identities with AcMNPV, at 84-98\%, suggesting that a similar mechanism for late gene transcription might occur in the Baculoviridae group.

\section{DNA replication genes}

A major group of conserved genes involved in DNA replication was described previously [5, 21, 24, 47]. AcMNPV and OpMNPV contain 5 genes that are essential for transient DNA replication (ie-1, lef-1, lef-2, lef-3 and helicase) and 5 non-essential ones that stimulate transient DNA replication genes (dna-pol, p35, ie-2, 
Table 2 Baculovirus gene category in TraeNPV

\begin{tabular}{|c|c|c|c|c|c|c|c|}
\hline \multirow{2}{*}{$\frac{\text { Category }}{\text { Replication genes (19) }}$} & \multicolumn{7}{|c|}{ Genes in TraeNPV } \\
\hline & $38.7 K(T a 5)$ & lef-1 & $d b p 1$ & gta & pena-a & $p c n a-b$ & DNA-pol \\
\hline & lef-3 & helicase & p35 & me53 & $i e-0$ & $i e-1$ & $i e-2$ \\
\hline & $38.7 K(T a 138)$ & lef-2 & pe38 & $l e f-7^{a}$ & $39 K^{a}$ & & \\
\hline \multirow[t]{2}{*}{ Transcription genes (13) } & lef-6 & lef-11 & p47 & lef-12 & lef-8 & lef-10 & lef-9 \\
\hline & $v \mid f-1$ & lef-4 & lef-5 & $l e f-7^{a}$ & pe38 a & $39 K^{\mathrm{a}}$ & \\
\hline \multirow[t]{5}{*}{ Structural genes (35) } & polh & orf1629 & pif-2 & efp/ld130 & odv-e66 & ac53 & vp1054 \\
\hline & fp25K & desmop & ac68 & ac78 & $g p 41$ & $a c 81$ & vp91/p95 \\
\hline & vp39 & p33 & odv-e25 & p6.9 & $p 40$ & vp80 & pif-3 \\
\hline & pif-1 & gp64 & p24 & gp16 & pp34 & p10 & p74 \\
\hline & p49 & odv-e18 & odv-e27 & odv-e56 & ac109 & ac16 & $19 \mathrm{kda}$ \\
\hline \multirow[t]{2}{*}{ Auxiliary genes (14) } & ptp & egt & alk-exo & $v$-cath & arif-1 & sod & fgf \\
\hline & ubiquitin & pkip & p26 & t/p20 & p12 & cg30 & chitinase \\
\hline
\end{tabular}

Genes are categorized based on their functions during virus replication

${ }^{\mathrm{a}} \mathrm{Gene}$, which is involved in multiple funcitons

lef-7, and pe38) [48-50]. These 10 genes are all present in the TraeNPV genome (Table 2). Six of these 10 genes (ie-1, lef-1, lef-2, lef-3, helicase and dna-pol) have previously been reported as essential DNA replication factors for baculoviruses, indicating that baculoviruses share a common DNA replication mechanism [50].

The other DNA replication genes, such as single-stranded DNA-binding protein $(d b p 1)$ and immediate-early gene (me53), which have been implicated in DNA replication, were also found in TraeNPV (Table 2) [51]. During viral infections, the host cell RNA polymerase II is often transactivated by genes such as $i e-0, i e-1, i e-2$ and $p e 38$. These genes are conserved relative to those of AcMNPV (84-98\%); however, a small variant form of the IE-2 protein was found between TraeNPV and other closely related NPVs (Fig. 7). Although the TraeNPV IE-2 amino acid sequence shared 92\% identity with that of the AcMNPV IE-2, the serine-rich and proline/glutamine-rich domains involved in activating a subset of early baculovirus promoters by AcMNPV IE-2 have a short deletion in the TraeNPV sequence (Fig. 7) [52]. A RING finger domain, which is required for cell cycle arrest, E3 ubiquitin ligase activity, and nuclear focus association; and a predicted coiled-coil region (coiled-coil-II), which is involved in self-interaction and association with nuclear foci, were strongly conserved in TraeNPV IE-2 and AcMNPV [53-56].

The TraeNPV genome encodes two PCNA proteins (Ta40 and Ta41), and both proteins had low shared amino acid identities with AcMNPV (53 and 36\%). Further investigation revealed that a single DNA base deletion resulted in two truncated forms of PCNA proteins, while a proliferating cell nuclear antigen PCNA protein may be involved in viral DNA replication, DNA recombination or DNA repair, but not the essential function of DNA replication, suggesting that the side effect of DNA replication may present differences between different viral species and hosts $[57,58]$.

\section{Genes with auxiliary functions}

Auxiliary genes are not essential for viral replication, but they provide a selective advantage for increasing the virus production/survival at either the cellular or organismal level [21]. A total of eighteen auxiliary genes have homologues in TraeNPV (Table 2). These auxiliary genes in TraeNPV were $90-100 \%$ identical in terms of amino acid sequences compared to those of AcMNPV, except for alk-exo and arif-1. The TraeNPV alk-exo was $81 \%$ identical to AcMNPV and its arif-1 was $72 \%$ identical to that of AcMNPV. According to the analysis, the lower shared identities were caused by amino acid length variations. The arif-1, which is involved in the sequential rearrangement of the actin cytoskeleton, is found only in NPVs [59]. Therefore, it may contribute to the morphological differences between different NPV and GV-infected cells.

\section{Homologous regions ( $h r s)$}

Homologous regions ( $h r s)$ is one of the feature found in most baculovirus genomes and locate in multiple sites in the genomes [60]. The structure of each $h r$ contains a palindrome, which is flanked by direct repeats. Hrs function as origins of NPVs and GVs replication [61] and also serve as RNA polymerase II-mediated transcription enhancers in early baculovirus promoters in NPVs [62] Recently, it has been reported that no single homologous repeat region is essential for the DNA replication of AcMNPV [63].

The TraeNPV genome contained eight homologous repeat regions ( $h r 1, h r 2, h r 3, h r 4, h r 5, h r 6, h r 7$ and $h r 8)$ 


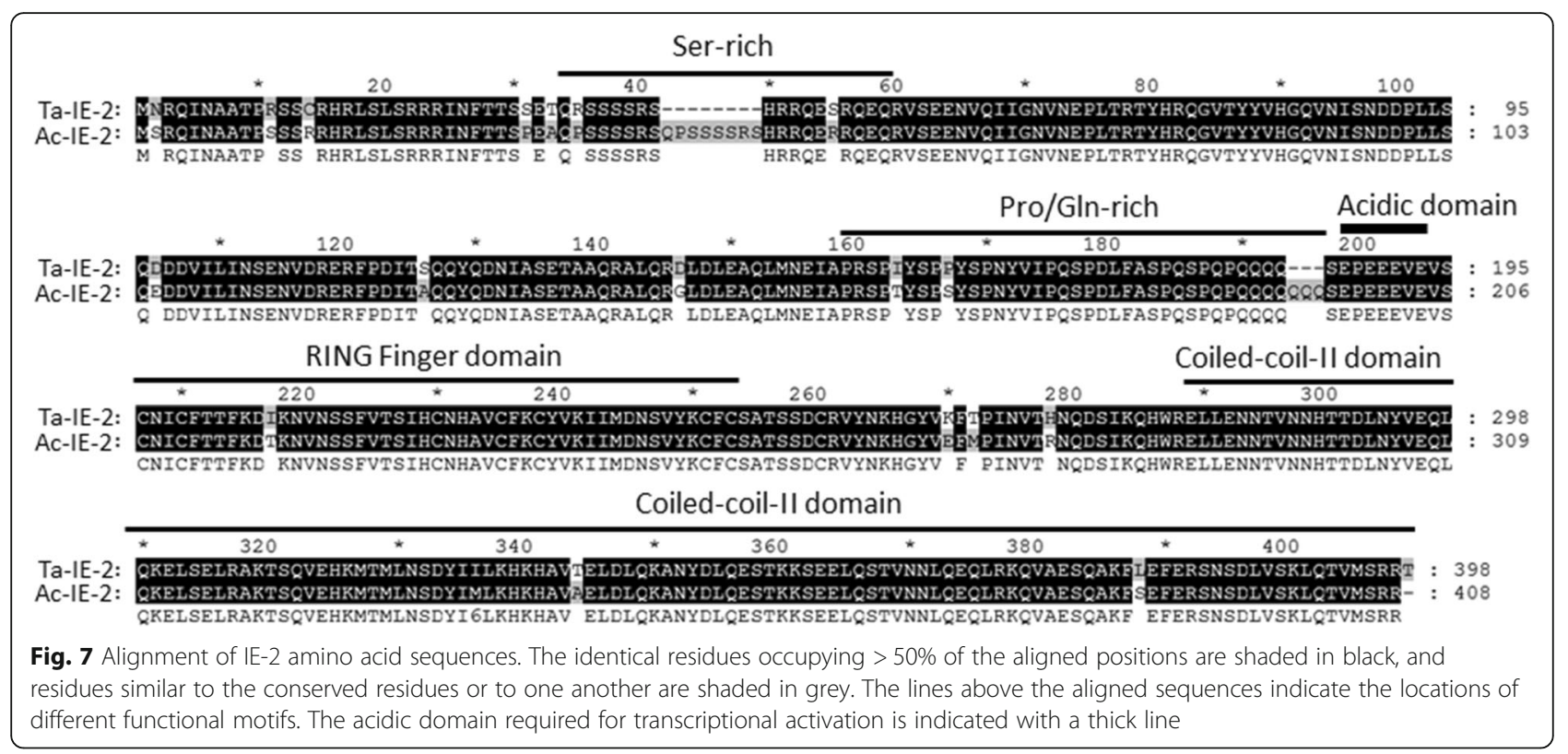

that included one to eight palindrome repeats for a total of 30 repeats (Fig. 8a and c) and accounted for $0.72 \%$ of the genome. Similar to the AcMNPV palindrome sequence [9], the TraeNPV $h r$ palindrome consensus GHKTTACRAGTAGAATTCTACDNGTAAHVC shows a 23/30 matched palindrome (Fig. 8b) and the palindromic consensus sequence included seven highly variable positions (Fig. 8b). All the nucleotides in the palindrome were conserved, except for the twenty-second nucleotide. In addition, the LdMNPV consensus $h r$ palindrome shared $43.3 \%$ of its sequence identity with the TraeNPV consensus $h r$ sequence (Fig. 8b). The genomic positions of the TraeNPV regions $h r 1-h r 8$ were conserved with the genomic positions of AcMNPV [9]; however, a lack of AcMNPV hr2- $a$ was found in the TraeNPV genome (Fig. 8c).

\section{Baculovirus repeated ORFs (bro genes)}

A striking feature of most lepidopteran and dipteran NPVs sequenced to date and in some of the GVs is the presence of one to 16 copies of bro genes. Typically, bro genes are highly conserved, repetitive and widely distributed amongst insect DNA viruses [64]. The function of these genes is unclear, but they have been shown to bind to DNA [65]. These genes have also been found to be associated with the viral genome rearrangement regions [66]. During the process of baculovirus replication, the viruses that synthesize mRNAs in the nucleus and this mRNA should be exported to the cytoplasm, while some viral proteins produced in the cytoplasm must be imported into the nucleus. It was demonstrated that the $\mathrm{BRO}$ proteins of BmNPV play a role in the function of the nucleocytoplasmic shuttling proteins that utilize the CRM1-mediated nuclear export pathway [67].

TraeNPV contained two bro genes, which were named bro- $a$ and bro- $b$ based on their order in the genome (Fig. 1; Additional file 1: Table S2). Most BROs contained a core sequence of 41 aa at the $\mathrm{N}$-terminal half and several different domains throughout the sequence. The bro gene family has been divided into four groups based on the similarity of those domains [12]. Both of the TraeNPV bro genes, namely Ta-bro-a (Ta141) and - $b$ (Ta142) (which were homologues of Bm-bro- $d$ ) belong to group III. Moreover, two TraeNPV bro genes encoded small fragments of truncated protein (234 aa and 92 aa). It has been reported that mutations in the leucine-rich region of $\mathrm{Bm}-\mathrm{BRO}$ proteins resulted in the nuclear accumulation of transiently expressed proteins; however, the mutant Bm-BRO-D with an altered nuclear export signal (NES) did not show nuclear accumulation in the infected cells due to a reduction in RNA synthesis [67], suggesting that the truncated $\mathrm{BRO}$ protein in TraeNPV may share a similar function as that of Bm-BRO-D.

\section{Genes involved in host range determination}

Baculoviruses usually showed high specificity to a few, or even individual, insect species $[68,69]$. For this reason, a variety of efforts has been made to understand the baculoviral genes that are related to their host range. Many viruses encode a variety of proteins related to the host range; AcMNPV is the most widely researched member of the Baculoviridae. AcMNPV contains several genes that involved in host range determination, including $p 143$ (helicase), hrf-1 (host range factor 1), hcf-1 (host 


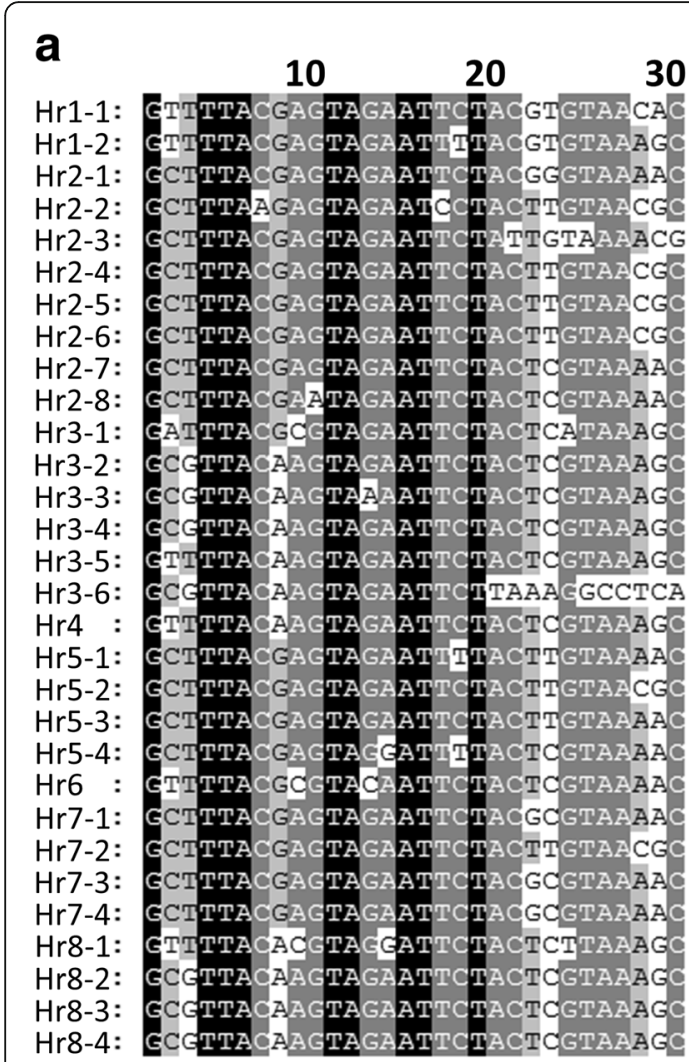

b

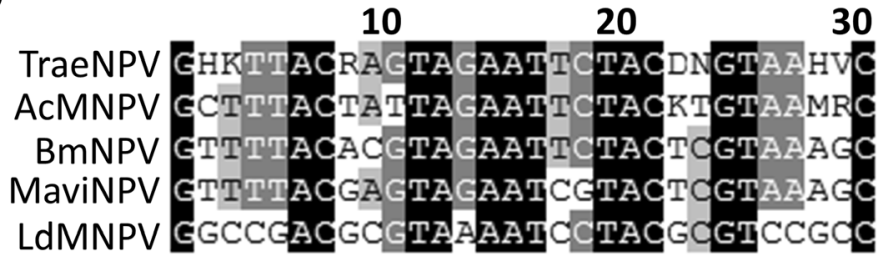

C

TraeMNPV

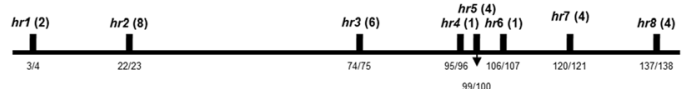

AcMNPV

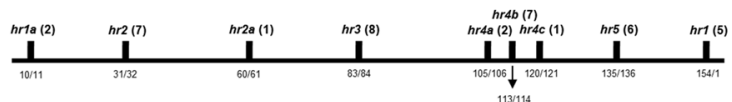

BmNPV

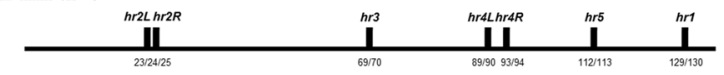

MaviNPV

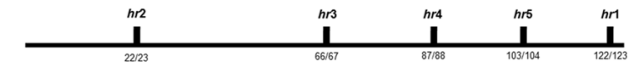

LdMNPV

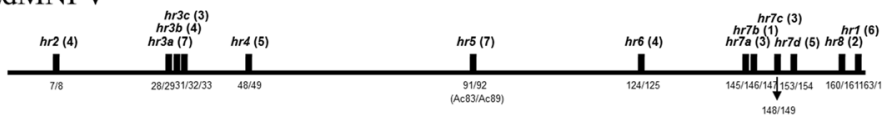

Fig. 8 Comparison of TraeNPV hr. palindromes with (a) each hr. palindrome, which was identified from the TraeNPV genome; and (b) palindrome consensus sequences from other baculoviruses. The alignment of the consensus hr. palindrome from TraeNPV, AcMNPV, BmNPV, MaviNPV and LdMNPV; and (c) a comparison of the genomic context of the hrs and hr. locations relative to the homologous ORFs between TraeNPV, AcMNPV, BmNPV, MaviNPV and LdMNPV in the linearized genomes. The ORFs flanking the hrs: below the line. Grey rectangles: the major inserts relative to AcMNPV and the ORFs within the inserts are shown above the line. For consistency, all the linearized genomes start with polh, but the hrs and ORF numbers remain the same as in the original papers

cell-specific factor 1), ie-2 and p35 [69-72]. Of these genes, $p 35$ and iap (inhibitor of apoptosis), are two major families of anti-apoptosis genes, which are commonly found in baculovirus genomes [73, 74].

The inhibition of vary caspase pathways by the $p 35$ and its homologue $p 49$ have been demonstracted [75]. The $p 35$ and $p 49$ are found in few sequenced baculoviruses, such as AcMNPV and Spodoptera litura MNPV (SpltMNPV) [9, 76]. For another anti-apoptosis gene family, the anti-apoptotic inhibition of IAP proteins has been demonstrated either directly or indirectly during baculovirus infection in permissive cells or heterogeneous insect cells in AcMNPV, Anticarsia gemmatalis MNPV (AgMNPV), Cydia pomonella granulovirus (CpGV), Epiphyas postvittana NPV (EppoNPV), Helicoverpa armigera NPV (HearNPV), Hyphantria cunea NPV (HycuNPV), Leucania separata MNPV (LeseMNPV), Orgyia pseudotsugata MNPV (OpMNPV), S. littoralis NPV (SpliNPV) and LyxyMNPV [75, 77-88]. Similar to AcMNPV, in the TraeNPV genome, $p 35$ (Ta119) and two iaps, iap1 (Ta18) and iap2 (Ta62), were identified. The amino acid identities of these three proteins are 97, 95 and 84\% shared with those of AcMNPV; it is speculated that these proteins might share similar activities in the host cells.

Recently, ld-apsup (ld109), a novel gene that inhibits apoptosis in LdMNPV-infected Ld652Y cells, was identified and its anti-apoptotic activities and mechanism were demonstrated $[89,90]$. According to a survey of the genome data, AcMNPV (Ac112-113) and other 17 baculoviruses contained apsup homologue genes in their genomes [89]. Interestingly, a lack of Ac112-113 was found in the TraeNPV genome (Table 1), and more extensive experiments may be performed to investigate the host range issue.

\section{TraeNPV truncated and duplicate genes}

There were three truncated ORFs (pcna-a/pcna-b, he65- $a /$ he $65-b$ and bro-a/bro- $b$ ) and one duplicated ORF (38.7 $\mathrm{K}$ in the Ta5 and Ta138 locations) located in the TraeNPV genome. All of the truncated ORFs showed low shared identities with their homologues in 
AcMNPV. For pcna-alpcna-b (Ta40/Ta41), the amino acid identities are 53 and $36 \%$ shared, respectively, compared to Ac49; 4 and 12\% in he65-a/he65-b (Ta93/Ta94) compared to Ac105; and 56 and $16 \%$ in bro-a/bro-b (Ta140/Ta141) compared to Ac2. For these truncated genes, the nucleotide deletions leading to the introduction of stop codons were found in both pcna-a/pcna-b (Ta40/Ta41) and bro-a/bro-b (Ta140/Ta141). For pcna-a/pcna-b (Ta40/Ta41), a one-bp deletion was found in the downstream $398 \mathrm{bp}$ (+398 bp) of ac-pcna; this deletion resulted in the introduction of a stop codon (TGA) in the + 434 bp, and thus a second pcna-b start codon was found between $+436 \mathrm{bp}$ and the end of this gene. In bro-a/bro-b (Ta140/Ta141), a seven-bp deletion was found $222 \mathrm{bp}$ downstream (+ $222 \mathrm{bp})$ of ac-bro, and this deletion resulted in the introduction of a stop codon (TGA) in the $-284 \mathrm{bp}$. Thus, a second bro- $b$ start codon was found between $+283 \mathrm{bp}$ and the end of this gene. For he65-a/he65-b (Ta93/Ta94), instead of the full-length he65 (553 aa) in AcMNPV, TraeNPV encoded two smaller proteins, he65- $a$ (58 aa) and he65- $b$ (72 aa). The HE65 protein is one of the RNA ligase families, and it acts as an early transcription gene involved in RNA replication, transcription and modification as well as in G-actin localization in the nucleus during AcMNPV cell infection. Although truncated he65 was found in the genome, it is considered a nonessential protein for AcMNPV and BmNPV [91, 92].

One pair of genes $(\mathrm{Ta} 5 / \mathrm{Ta} 138)$ was identified as duplicated homologues of $38.7 \mathrm{~K}$ in the TraeNPV genome. This duplicate gene (Ta138) showed low shared identities with the homologues of AcMNPV (15\%).

\section{Unique TraeNPV ORFs}

Two genes are unique in the TraeNPV genome, including Ta75 and Ta139 (Fig. 1; Additional file 1: Table S2). These unique ORFs were small in size (55-60 aa). Both Ta75 and Ta139 had no any baculovirus homologue and no significant BLAST database hit. However, the promoter region should be predicted in the future to evaluate the transcriptional contribution to TraeNPV.

\section{Comparison of TraeNPV to AcMNPV}

Based on the sequence analysis, TraeNPV was highly similar to AcMNPV. The phylogenetic analysis indicated that TraeNPV belonged to Alphabaculovirus Group I. However, there were still some distinctions in the genomic features and gene content between these two viruses. The most significant difference between TraeMNPV and AcMNPV was that the TraeNPV genome is $8417 \mathrm{bp}$ smaller than the AcMNPV genome (133,894 bp) and it contains 15 fewer ORFs (Table 1), while the TraeNPV genome contained two ORFs that were not found in the AcMNPV genome (Additional file 1: Table S2). Moreover, according to the data on the in silico restriction enzyme fragment length polymorphism (in silico RFLP) pattern using BamHI, TraeNPV showed a different pattern compared to that of AcMNPV (Additional file 4: Figure S3). The AcMNPV genome contains 15 ORFs, which were not found in the TraeNPV genome. Two genes that encode HCF-1 and APSUP were described as the host range determination factors in baculoviruses [89, 90]. It has been demonstrated that the AcMNPV HCF-1 protein is an essential viral factor for the productive NPV infection of TN-368 cells [93, 94]. Recently, a novel anti-apoptotic protein, APSUP, was identified in LdMNPV [95]; moreover, it has been demonstrated that the full-length Ld-Apsup could work against the apoptosis of Ld652Y cells induced by exposure to actinomycin D and UV and could interact with Ld-Dronc to prevent cells from undergoing apoptosis. The baculovirus host range likely involves a complicated array of viral and cellular factors. Based on the data from the genomic analysis, a lack of Ac112-113 was found in the TraeNPV genome (Table 1), and more extensive experiments may be performed to uncover more evidence regarding the host range issue.

There were 142 ORFs in common between TraeNPV and AcMNPV, and their order is mostly identical. However, several of these ORFs had different lengths, as shown in Fig. 6. These genes included arif-1, IAP2, $v p 91 / p 95, p p 34$, alk-exo, odv-e18 and ie-2 as well as other genes with unassigned functions. Moreover, three pairs of truncated genes were found in the TraeNPV genome, namely, pcna-a/pcna-b, he65-a/he65-b and bro-a/bro- $b$. These truncated genes also showed amino acid length variations between TraeNPV and AcMNPV (Fig. 6). The hrs of TraeNPV are similar to those of AcMNPV in terms of their position, numbers and orientations, while there was no $h r 2 a$ in TraeNPV. The gene content, ORF length and $h r$ are possible candidates for regulators of the different virulence levels between two closely relative species [67], which might be the case for TraeNPV and AcMNPV.

\section{Conclusions}

In conclusion, TraeNPV showed a high degree of collinearity and shared sequence identity with AcMNPV. However, these two viruses showed different host ranges and geographical distribution. To date, TraeNPV has only been isolated from $T$. aeacus, which is a native butterfly species under conservation in Taiwan. Furthermore, although the genome sequence analysis revealed that TraeNPV lacks 15 homologous genes from AcMNPV, TraeNPV gained two novel unique genes. Interestingly, there were two host range determination genes, $h c f-1$ and apsup, in AcMNPV (and also in other 
alphabaculoviruses) that were not found in TraeNPV. These findings were very interesting and worthy of further studies to collect more evidence about the host range issue. Based on our analytical data, TraeNPV would be clarified as a new NPV species, which has defective AcMNPV genomic features. The lack of $h c f-1$ and apsup in the genomic sequence data for TraeNPV could provide useful information for understanding the baculoviral host ranges and for gaining evolutionary insights.

\section{Methods}

\section{Viral DNA extraction and DNA sequencing}

Diseased $T$. aeacus larvae samples were homogenized in $1.7 \mathrm{~mL}$ microcentrifuge tubes and then examined under a light microscope for viral occlusion bodies (OBs). To obtain the OBs, the samples were centrifuged at $14,000 \times g$ at $4{ }^{\circ} \mathrm{C}$ for $10 \mathrm{~min}$ and the supernatants were removed. The pellets were washed in $1 \times$ TE buffer $(10$ $\mathrm{mM}$ Tris- $\mathrm{HCl}$, and $1 \mathrm{mM}$ EDTA, $\mathrm{pH} 7.6)$ and centrifuged three times at $14,000 \times g$ at $4{ }^{\circ} \mathrm{C}$ for $10 \mathrm{~min}$. The pellets were then resuspended in $1 \times \mathrm{TE}$ buffer with a final concentration of $1 \%(\mathrm{w} / \mathrm{v})$ SDS and then incubated with proteinase $\mathrm{K}(0.25 \mathrm{mg} / \mathrm{ml})$ at $56^{\circ} \mathrm{C}$ for $3 \mathrm{~h}$. The total DNA (including the host and viral DNA) was extracted using previously published methods [96]. A sequencing library was prepared following the standard protocol from the NEBNext Ultra II DNA Library Prep Kit for Illumina (NEB) and sequenced with an Illumina MiSeq sequencer with paired-end (PE) technology for $2 \times 300$ bp.

\section{Data pre-processing and bioinformatics analysis}

The total PE reads were conducted for sequencing adapter identification and then trimmed by cutadapt [97]. Ambiguous bases and bases with lower quality values were removed by PRINseq [98] from either the 5 ' - or 3 '-end. The final high quality reads were selected using NGS QC Toolkit [99] with the default parameters (Additional file 1: Table S4). These trimmed reads were then subjected to genome assembly and annotation by bioinformatics analysis (Additional file 5: Figure S4).

The strategy for TraeNPV genome assembly is to employ the longer paired-end (PE) reads. The genome assembly approach used in this study is reference-guided assembly, with benefits from the reference organism. The reference species is identified as the top-ranked individual with the highest read count by mapping PE reads against the collection of viral genomes from NCBI GenBank. MIRA [100], one of the reference-guide assembly types, maps sequencing reads against reference species to generate the genome sequence of target species. Gap elimination was applied using an in-house script programme by mapping the quality PE reads and contigs iteratively until convergence was reached.
Contigs are the joined paired-end reads found by using COPE [101] and assembled contigs were found by de novo assembly, with SOAPdenovo [102]. Draft genome gap filling and gene coding region validation were performed by Sanger sequencing to complete the final genome and gene annotation, respectively. The designed primer sets for PCR validation are listed in Additional file 1: Tables S5 and S6.

The genome annotations were performed with both NCBI ORF finder (https://www.ncbi.nlm.nih.gov/orffinder/) and Glimmer [103] to identify the open reading frames in the genome. Repetitive sequence regions were detected by RepeatMasker (http://www.repeatmasker.org/). CD-HIT and BLASTN in the NCBI BLAST package were used to identify the correctness of the predicted genes and the corresponding sequence identities. A circular map of the viral genome was generated by CGView [104].

\section{Phylogenetic analysis}

The phylogenetic tree was inferred from a data set of concatenated amino acid sequences from the 37 baculovirus core genes $[5,16]$ of the 77 baculoviruses that were completely sequenced at the time of analysis (Additional file 1: Table S3). A maximum likelihood (ML) analysis was performed using MEGA version 7.0 [105]. Culex nigripalpus NPV (CuniNPV) [106] was selected as the out-group. A bootstrap analysis was performed to evaluate the robustness of the phylogenies using 100 replicates for ML analysis.

\section{Comparative genomic analysis}

Both the whole genome and all the putative ORFs of TraeNPV were subjected to a comparative genomic analysis with 4 alphabaculoviruses (3 group I NPVs and 1 group II NPVs) and 1 betabaculovirus using the CGView Comparison Tool (CCT) [107]. Moreover, the multiple alignment of conserved genomic sequence with rearrangements was performed by Mauve [108].

\section{Additional files}

\footnotetext{
Additional file 1: Table S1. Characteristics of current sequenced baculovirus genomes. ${ }^{1} \mathrm{n} / \mathrm{a}=$ no information is available in either the paper or GenBank file. ${ }^{2}$ The GenBank file with accession number KX1513952 is not available at the GenBank website. ${ }^{\S}$ Virus name printed in bold were used for further comparison. Table S2. ORFs predicted in the TraeNPV genome. Table S3. ORF numbers of the 37 baculovirus core genes from 77 baculoviruses. Table S4. Sequencing library statistics. Table S5. Primer sets for validating the genome assembly and gap filling ${ }^{*}$ A total of 103 primer sets were used in this study. The average amplicon size is ca. $1150 \mathrm{bp}$ and the re-sequenced coverage is ca. 1. Table S6. Primer sets for validating the gene coding regions. (XLSX $99 \mathrm{~kb}$ )

Additional file 2: Figure S1. Genome density of TraeNPV compared to 78 sequenced baculoviruses. Genome density = number of ORFs/genome size; ratio of genome density = relative genome density to that of TraeNPV. The number behind the column represents the order of the
} 
relative genome density among 79 sequenced baculoviruses. (TIFF 2612 $\mathrm{kb})$

Additional file 3: Figure S2. Heat map of the genome. The heat map identity of the genomes from the species AcMNPV, BmNPV, MaviMNPV, LdMNPV and CpGV (from the outside to the inside) compared to the orthologous ORFs in TraeNPV. The darker the red is, the higher the correlated genomic fragment identity. (TIFF $1135 \mathrm{~kb}$ )

Additional file 4: Figure S3. In silico Restriction Fragment Length Polymorphism (in silico RFLP) pattern based on the whole genomic sequences of TraeNPV and AcMNPV as cut with BamHI restriction enzyme. (TIFF $179 \mathrm{~kb}$ )

Additional file 5: Figure S4. Flowchart of bioinformatics analysis. (TIFF $711 \mathrm{~kb})$

\section{Abbrevations}

NGS: Next-generation sequencing; NPV: Nucleopolydedrovirus; ORF: Open reading frame; $P E$ : Paired end

\section{Acknowledgements}

We are grateful to the National Center for High-performance Computing for computer time and facilities. The authors also thank Prof. Chung-Hsiung Wang at Natioanl Taiwan University for giving us valuable and constructive suggestion on manuscript.

\section{Funding}

This research was supported by Grant 106-2311-B-197-001- from the Ministry of Science and Technology (MOST), Taiwan and the project grant from Rural Development Administration, RDA, Republic of Korea. The funding bodies had no role in study design, data collection and analysis, decision to publish or preparation of the manuscript.

\section{Availability of data and materials}

GenBank accession number: MH077961.

The dataset used and/or analyzed during the current study are available from the corresponding author on reasonable request.

\section{Authors' contributions}

YFH and THC carried out the experiments and analysed these sequences. THC and YSN purified the virus genomic DNA. YSN, THC, YFH, ZTC, SJL, JCK, and JSK analysed sequences and confirmed the validation results. CHW, KPC, YFH and YSN carried out the design and draft of the manuscript. All authors read and approved the final manuscript.

\section{Ethics approval and consent to participate}

Not applicable.

\section{Consent for publication}

Not applicable.

\section{Competing interests}

The authors declare that they have no competing interests.

\section{Publisher's Note}

Springer Nature remains neutral with regard to jurisdictional claims in published maps and institutional affiliations.

\section{Author details}

${ }^{1}$ Genomics Research Center, Academia Sinica, Taipei, Taiwan. ${ }^{2}$ Department of Biotechnology and Animal Science, National Ilan University, Yilan, Taiwan. ${ }^{3}$ Department of Entomology, National Taiwan University, Taipei, Taiwan. ${ }^{4}$ Department of Agricultural Biology, College of Agriculture \& Life Sciences, Chonbuk National University, Jeonju, South Korea. ${ }^{5}$ Department of Entomology, National Chung Hsing University, Taichung, Taiwan.
Received: 31 July 2018 Accepted: 17 April 2019

Published online: 27 May 2019

\section{References}

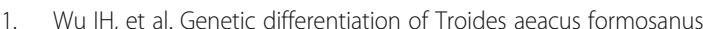
(Lepidoptera: Papilionidae), based on cytochrome oxidase I sequences and amplified fragment length polymorphism. Genetics. 2010;130(6):1018-24.

2. Haugum J, Low AM. A monograph of the birdwing butterflies, the systematics of Ornithoptera, Troides and related genera: vol. 2, the genera Trogonoptera, Ripponia and Troides. Klampenborg: Scandinavian SciencePress; 1985.

3. Collins NM, Morris MG. the IUCN red data book. In: Threatened swallowtail butterßies of the world. Gland and Cambridge: IUCN press; 1985.

4. Nai YS, et al. Biological Control of Pest and Vector Insects. Determination of Nucleopolyhedrovirus' Taxonomic Position. ed. Vonnie D.C Shields. Chapter 8. London: InTech; 2017

5. Jehle JA, et al. On the classification and nomenclature of baculoviruses: a proposal for revision. Arch Virol. 2006;151(7):1257-66.

6. Bulach DM, et al. Group II nucleopolyhedrovirus subgroups revealed by phylogenetic analysis of polyhedrin and DNA polymerase gene sequences. J Invertebr Pathol. 1999;73(1):59-73

7. Wang J, et al. Genome sequencing and analysis of Catopsilia Pomona nucleopolyhedrovirus: a distinct species in group I Alphabaculovirus. PLoS One. 2016:11(5):e0155134.

8. Jehle JA, et al. Molecular identification and phylogenetic analysis of baculoviruses from Lepidoptera. Virology. 2006:346(1):180-93.

9. Ayres MD, et al. The complete DNA sequence of Autographa californica nuclear polyhedrosis virus. Virology. 1994;202(2):586-605.

10. Gomi S, Majima K, Maeda S. Sequence analysis of the genome of Bombyx mori nucleopolyhedrovirus. J Gen Virol. 1999;80(Pt 5):1323-37.

11. Chen YR, et al. Genomic and host range studies of Maruca vitrata nucleopolyhedrovirus. J Gen Virol. 2008;89(Pt 9):2315-30.

12. Kuzio J, et al. Sequence and analysis of the genome of a baculovirus pathogenic for Lymantria dispar. Virology. 1999;253(1):17-34.

13. Luque T, et al. The complete sequence of the Cydia pomonella granulovirus genome. J Gen Virol. 2001;82(Pt 10):2531-47.

14. Vlak JM, Smith GE. Orientation of the genome of Autographa californica nuclear Polyhedrosis virus: a proposal. J Virol. 1982;41(3):1118-21.

15. Garcia-Maruniak A, et al. Sequence analysis of the genome of the Neodiprion sertifer nucleopolyhedrovirus. J Virol. 2004;78(13):7036-51.

16. Herniou EA, et al. The genome sequence and evolution of baculoviruses. Annu Rev Entomol. 2003:48:211-34.

17. McCarthy CB, Theilmann DA. AcMNPV ac143 (odv-e18) is essential for mediating budded virus production and is the 30th baculovirus core gene. Virology. 2008;375(1):277-91

18. Aragao-Silva CW, et al. The complete genome of a baculovirus isolated from an insect of medical interest: Lonomia obliqua (Lepidoptera: Saturniidae). Sci Rep. 2016:6:23127.

19. Garavaglia MJ, et al. The ac53, ac78, ac101, and ac103 genes are newly discovered core genes in the family Baculoviridae. J Virol. 2012:86(22):12069-79.

20. Kikhno I. Identification of a conserved non-protein-coding genomic element that plays an essential role in Alphabaculovirus pathogenesis. PLoS One 2014:9(4):e95322.

21. Hayakawa T, Rohrmann GF, Hashimoto Y. Patterns of genome organization and content in lepidopteran baculoviruses. Virology. 2000;278(1):1-12.

22. Herniou EA, et al. Use of whole genome sequence data to infer baculovirus phylogeny. J Virol. 2001:75(17):8117-26.

23. Harrison RL, Bonning BC. Comparative analysis of the genomes of Rachiplusia ou and Autographa californica multiple nucleopolyhedroviruses. J Gen Virol. 2003;84(Pt 7):1827-42.

24. Thumbi DK, et al. Comparative genome sequence analysis of Choristoneura occidentalis Freeman and C. rosaceana Harris (Lepidoptera: Tortricidae) alphabaculoviruses. PLoS One. 2013;8(7):e68968.

25. Faulkner $\mathrm{P}$, et al. Analysis of p74, a PDV envelope protein of Autographa californica nucleopolyhedrovirus required for occlusion body infectivity in vivo. J Gen Virol. 1997:78(Pt 12):3091-100.

26. Kuzio J, Jaques R, Faulkner P. Identification of p74, a gene essential for virulence of baculovirus occlusion bodies. Virology. 1989:173(2):759-63.

27. Olszewski J, Miller LK. Identification and characterization of a baculovirus structural protein, VP1054, required for nucleocapsid formation. J Virol. 1997;71(7):5040-50. 
28. Gross CH, Russell RL, Rohrmann GF. Orgyia pseudotsugata baculovirus p10 and polyhedron envelope protein genes: analysis of their relative expression levels and role in polyhedron structure. J Gen Virol. 1994;75(Pt 5):1115-23.

29. Williams GV, et al. A cytopathological investigation of Autographa californica nuclear polyhedrosis virus p10 gene function using insertion/ deletion mutants. J Gen Virol. 1989;70(Pt 1):187-202.

30. van Oers MM, et al. Functional domains of the p10 protein of Autographa californica nuclear polyhedrosis virus. J Gen Virol. 1993;74(Pt 4):563-74.

31. Lu A, Carstens EB. Nucleotide sequence and transcriptional analysis of the p80 gene of Autographa californica nuclear polyhedrosis virus: a homologue of the Orgyia pseudotsugata nuclear polyhedrosis virus capsidassociated gene. Virology. 1992;190(1):201-9.

32. Muller $\mathrm{R}$, et al. A capsid-associated protein of the multicapsid nuclear polyhedrosis virus of Orgyia pseudotsugata: genetic location, sequence, transcriptional mapping, and immunocytochemical characterization. Virology. 1990;176(1):133-44.

33. Russell RL, Funk CJ, Rohrmann GF. Association of a baculovirus-encoded protein with the capsid basal region. Virology. 1997;227(1):142-52.

34. Vialard JE, Richardson CD. The 1,629-nucleotide open reading frame located downstream of the Autographa californica nuclear polyhedrosis virus polyhedrin gene encodes a nucleocapsid-associated phosphoprotein. J Virol. 1993;67(10):5859-66.

35. IJkel WF, et al. A novel baculovirus envelope fusion protein with a proprotein convertase cleavage site. Virology. 2000;275(1):30-41.

36. Pearson MN, Groten C, Rohrmann GF. Identification of the lymantria dispar nucleopolyhedrovirus envelope fusion protein provides evidence for a phylogenetic division of the Baculoviridae. J Virol. 2000;74(13):6126-31.

37. Olszewski J, Miller LK. A role for baculovirus GP41 in budded virus production. Virology. 1997;233(2):292-301.

38. Whitford M, Faulkner P. A structural polypeptide of the baculovirus Autographa californica nuclear polyhedrosis virus contains O-linked N-acetylglucosamine. J Virol. 1992;66(6):3324-9.

39. Jehle JA. The mosaic structure of the polyhedrin gene of the Autographa californica nucleopolyhedrovirus (AcMNPV). Virus Genes. 2004;29(1):5-8.

40. Li L, Harwood SH, Rohrmann GF. Identification of additional genes that influence baculovirus late gene expression. Virology. 1999;255(1):9-19.

41. Lu A, Miller LK. Identification of three late expression factor genes within the 33.8- to 43.4-map-unit region of Autographa californica nuclear polyhedrosis virus. J Virol. 1994;68(10):6710-8.

42. Rankin C, Ooi BG, Miller LK. Eight base pairs encompassing the transcriptional start point are the major determinant for baculovirus polyhedrin gene expression. Gene. 1988;70(1):39-49.

43. Guarino LA, Summers MD. Functional mapping of Autographa california nuclear polyhedrosis virus genes required for late gene expression. J Virol. 1988;62(2):463-71.

44. McLachlin JR, Miller LK. Identification and characterization of vlf-1, a baculovirus gene involved in very late gene expression. J Virol. 1994;68(12):7746-56

45. Yang S, Miller LK. Control of baculovirus polyhedrin gene expression by very late factor 1. Virology. 1998;248(1):131-8.

46. Yang $S$, Miller LK. Activation of baculovirus very late promoters by interaction with very late factor 1. J Virol. 1999;73(4):3404-9.

47. Rapp JC, Wilson JA, Miller LK. Nineteen baculovirus open reading frames, including LEF-12, support late gene expression. J Virol. 1998;72(12):10197-206.

48. McDougal W, Guarino LA. The Autographa californica nuclear polyhedrosis virus p143 gene encodes a DNA helicase. J Virol. 2000;74(11):5273-9.

49. Kool M, et al. Replication of baculovirus DNA. J Gen Virol. 1995;76(Pt 9):2103-18.

50. Lu A, et al. Baculovirus DNA replication. In: Miller LKE, editor. The Baculoviruses; 1997.

51. Mikhailov VS, et al. Bombyx mori nucleopolyhedrovirus encodes a DNA-binding protein capable of destabilizing duplex DNA. J Virol. 1998; 72(4):3107-16

52. Yoo S, Guarino LA. Functional dissection of the ie2 gene product of the baculovirus Autographa californica nuclear polyhedrosis virus. Virology. 1994;202(1):164-72.

53. Prikhod'ko EA, Miller LK. Role of baculovirus IE2 and its RING finger in cell cycle arrest. J Virol. 1998;72(1):684-92.

54. Imai N, Matsumoto S, Kang W. Formation of Bombyx mori nucleopolyhedrovirus IE2 nuclear foci is regulated by the functional domains for oligomerization and ubiquitin ligase activity. J Gen Virol. 2005;86(Pt 3):637-44
55. Imai N, et al. Ubiquitin ligase activities of Bombyx mori nucleopolyhedrovirus RING finger proteins. J Virol. 2003;77(2):923-30.

56. Harrison RL, Lynn DE. Genomic sequence analysis of a nucleopolyhedrovirus isolated from the diamondback moth. Plutella xylostella Virus Genes. 2007;35(3):857-73

57. Kool M, et al. Identification of genes involved in DNA replication of the Autographa californica baculovirus. Proc Natl Acad Sci U S A. 1994;91(23):11212-6.

58. Pearson MN, Rohrmann GF. Characterization of a baculovirus-encoded ATPdependent DNA ligase. J Virol. 1998;72(11):9142-9.

59. Roncarati $R$, Knebel-Morsdorf $D$. Identification of the early actinrearrangement-inducing factor gene, arif-1, from Autographa californica multicapsid nuclear polyhedrosis virus. J Virol. 1997;71(10):7933-41.

60. Garcia-Maruniak A, Pavan OH, Maruniak JE. A variable region of Anticarsia gemmatalis nuclear polyhedrosis virus contains tandemly repeated DNA sequences. Virus Res. 1996;41(2):123-32.

61. Hilton S, Winstanley D. Identification and functional analysis of the origins of DNA replication in the Cydia pomonella granulovirus genome. J Gen Virol. 2007:88(Pt 5):1496-504.

62. Theilmann DA, Stewart S. Tandemly repeated sequence at the $3^{\prime}$ end of the IE-2 gene of the baculovirus Orgyia pseudotsugata multicapsid nuclear polyhedrosis virus is an enhancer element. Virology. 1992;187(1):97-106.

63. Carstens EB, Wu Y. No single homologous repeat region is essential for DNA replication of the baculovirus Autographa californica multiple nucleopolyhedrovirus. J Gen Virol. 2007;88(Pt 1):114-22

64. Bideshi DK, et al. Phylogenetic analysis and possible function of bro-like genes, a multigene family widespread among large double-stranded DNA viruses of invertebrates and bacteria. J Gen Virol. 2003:84(Pt 9):2531-44.

65. Zemskov EA, Kang W, Maeda S. Evidence for nucleic acid binding ability and nucleosome association of Bombyx mori nucleopolyhedrovirus BRO proteins. J Virol. 2000;74(15):6784-9.

66. Li L et al. Complete comparative genomic analysis of two field isolates of Mamestra configurata nucleopolyhedrovirus-a. J Gen Virol. 2005;86(Pt 1):91-105.

67. Kang W, Kurihara M, Matsumoto S. The BRO proteins of Bombyx mori nucleopolyhedrovirus are nucleocytoplasmic shuttling proteins that utilize the CRM1-mediated nuclear export pathway. Virology. 2006:350(1):184-91.

68. Morris TD, Miller LK. Promoter influence on baculovirus-mediated gene expression in permissive and nonpermissive insect cell lines. J Virol. 1992;66(12):7397-405.

69. Wu C, et al. Generating a host range-expanded recombinant baculovirus. Sci Rep. 2016:6:28072

70. Everett $H$, McFadden G. Viruses and apoptosis: meddling with mitochondria. Virology. 2001;288(1):1-7

71. Thiem SM, Cheng X-W. Baculovirus host-range. Virologica Sinica. 2009;24(5): 436-57.

72. Clem RJ. In: Miller LK, editor. The Baculoviruses The Viruses. US: Springer; 1997.

73. Clem RJ, Fechheimer M, Miller LK. Prevention of apoptosis by a baculovirus gene during infection of insect cells. Science. 1991;254(5036):1388-90.

74. Clem RJ. Baculoviruses and apoptosis: a diversity of genes and responses. Curr Drug Targets. 2007:8(10):1069-74.

75. Crook NE, Clem RJ, Miller LK. An apoptosis-inhibiting baculovirus gene with a zinc finger-like motif. J Virol. 1993:67(4):2168-74.

76. Du Q, et al. Isolation of an apoptosis suppressor gene of the Spodoptera littoralis nucleopolyhedrovirus. J Virol. 1999:73(2):1278-85.

77. Birnbaum MJ, Clem RJ, Miller LK. An apoptosis-inhibiting gene from a nuclear polyhedrosis virus encoding a polypeptide with Cys/his sequence motifs. J Virol. 1994;68(4):2521-8.

78. Ikeda M, Yanagimoto K, Kobayashi M. Identification and functional analysis of Hyphantria cunea nucleopolyhedrovirus iap genes. Virology. 2004;321(2):359-71.

79. Carpes MP, et al. The inhibitor of apoptosis gene (IAP-3) of Anticarsia gemmatalis multicapsid nucleopolyhedrovirus (AgMNPV) encodes a functional IAP. Arch Virol. 2005:150(8):1549-62

80. Kim YS, et al. Identification and functional analysis of LsMNPV anti-apoptosis genes. J Biochem Mol Biol. 2007:40(4):571-6.

81. Liang C, et al. Functional analysis of two inhibitor of apoptosis (iap) orthologs from Helicoverpa armigera nucleopolyhedrovirus. Virus Res. 2012;165(1):107-11.

82. Vilaplana L, O'Reilly DR. Functional interaction between Cydia pomonella granulovirus IAP proteins. Virus Res. 2003;92(1):107-11. 
83. Means JC, Muro I, Clem RJ. Silencing of the baculovirus Op-iap3 gene by RNA interference reveals that it is required for prevention of apoptosis during Orgyia pseudotsugata M nucleopolyhedrovirus infection of Ld652Y cells. J Virol. 2003;77(8):4481-8.

84. Maguire T, et al. The inhibitors of apoptosis of Epiphyas postvittana nucleopolyhedrovirus. J Gen Virol. 2000;81(Pt 11):2803-11.

85. Yan F, et al. Functional analysis of the inhibitor of apoptosis genes in Antheraea pernyi nucleopolyhedrovirus. J Microbiol. 2010;48(2):199-205.

86. Wu YL, et al. Heliothis zea nudivirus 1 gene hhi1 induces apoptosis which is blocked by the Hz-iap2 gene and a noncoding gene, pag1. J Virol. 2011:85(14):6856-66.

87. Zeng $X$, et al. Functional analysis of the Autographa californica nucleopolyhedrovirus IAP1 and IAP2. Sci China C Life Sci. 2009;52(8):761-70.

88. Nai YS, et al. Genomic sequencing and analyses of Lymantria xylina multiple nucleopolyhedrovirus. BMC Genomics. 2010;11:116

89. Yamada $\mathrm{H}$, et al. Identification of a novel apoptosis suppressor gene from the baculovirus Lymantria dispar multicapsid nucleopolyhedrovirus. J Virol. 2011:85(10):5237-42.

90. Yamada $\mathrm{H}$, et al. Novel apoptosis suppressor Apsup from the baculovirus Lymantria dispar multiple nucleopolyhedrovirus precludes apoptosis by preventing proteolytic processing of initiator caspase Dronc. J Virol. 2013:87(23):12925-34.

91. Ohkawa T, Rowe AR, Volkman LE. Identification of six Autographa californica multicapsid nucleopolyhedrovirus early genes that mediate nuclear localization of G-actin. J Virol. 2002;76(23):12281-9.

92. Krejmer $\mathrm{M}$, et al. The genome of Dasychira pudibunda nucleopolyhedrovirus (DapuNPV) reveals novel genetic connection between baculoviruses infecting moths of the Lymantriidae family. BMC Genomics. 2015;16:759

93. Tachibana A, et al. HCF-1 encoded by baculovirus AcMNPV is required for productive nucleopolyhedrovirus infection of non-permissive Tn368 cells. Sci Rep. 2017;7(1):3807.

94. Lu A, Miller LK. Species-specific effects of the hcf-1 gene on baculovirus virulence. J Virol. 1996;70(8):5123-30.

95. Li HF, et al. Two maternal origins of Chinese domestic goose. Poult Sci. 2011;90(12):2705-10

96. Wang $\mathrm{CH}$, et al. Continuous cell line from pupal ovary of Perina nuda (Lepidoptera: Lymantriidae) that is permissive to nuclear Polyhedrosis virus from P. nuda. J Invertebr Pathol. 1996;67(3):199-204.

97. Martin M. Cutadapt removes adapter sequences from high-throughput sequencing reads. EMBnet J. 2011;17(1). Next Generation Sequencing Data Analysis 2011.

98. Schmieder R, Edwards R. Quality control and preprocessing of metagenomic datasets. Bioinformatics. 2011;27(6):863-4.

99. Patel RK, Jain M. NGS QC toolkit: a toolkit for quality control of next generation sequencing data. PLoS One. 2012;7(2):e30619.

100. Chevreux B, et al. Using the miraEST assembler for reliable and automated mRNA transcript assembly and SNP detection in sequenced ESTs. Genome Res. 2004;14(6):1147-59.

101. Liu B, et al. COPE: an accurate k-mer-based pair-end reads connection too to facilitate genome assembly. Bioinformatics. 2012;28(22):2870-4.

102. Luo $R$, et al. SOAPdenovo2: an empirically improved memory-efficient short-read de novo assembler. Gigascience. 2012;1(1):18.

103. Salzberg SL, et al. Microbial gene identification using interpolated Markov models. Nucleic Acids Res. 1998;26(2):544-8.

104. Stothard P, Wishart DS. Circular genome visualization and exploration using CGView. Bioinformatics. 2005;21(4):537-9.

105. Kumar S, Stecher G, Tamura K. MEGA7: molecular evolutionary Genetics analysis version 7.0 for bigger datasets. Mol Biol Evol. 2016;33(7):1870-4.

106. Afonso $\mathrm{CL}$, et al. Genome sequence of a baculovirus pathogenic for Culex nigripalpus. J Virol. 2001;75(22):11157-65.

107. Grant JR, Arantes AS, Stothard P. Comparing thousands of circular genomes using the CGView comparison tool. BMC Genomics. 2012;13:202.

108. Darling AC, et al. Mauve: multiple alignment of conserved genomic sequence with rearrangements. Genome Res. 2004;14(7):1394-403.

\section{Ready to submit your research? Choose BMC and benefit from:}

- fast, convenient online submission

- thorough peer review by experienced researchers in your field

- rapid publication on acceptance

- support for research data, including large and complex data types

- gold Open Access which fosters wider collaboration and increased citations

- maximum visibility for your research: over $100 \mathrm{M}$ website views per year

At BMC, research is always in progress.

Learn more biomedcentral.com/submissions 